\title{
Immune response after an experimental intramammary challenge with killed Staphylococcus aureus in cows and heifers vaccinated and not vaccinated with Startvac, a polyvalent mastitis vaccine
}

\author{
S. Piepers, ${ }^{* 1}$ A. Prenafeta, $†$ J. Verbeke, ${ }^{*}$ A. De Visscher, ${ }^{*}$ Ricard March, $\dagger$ and S. De Vliegher* \\ ${ }^{*}$ M-team \& Mastitis and Milk Quality Research Unit, Department of Reproduction, Obstetrics and Herd Health, Faculty of Veterinary Medicine, \\ Ghent University, Merelbeke 9820, Belgium \\ †Research and Development Department, Hipra Scientific, Secondary Logistical Unit, 17170 Amer, Girona, Spain
}

\begin{abstract}
An experimental trial was conducted to explore the effect of vaccination with a polyvalent vaccine against mastitis (Startvac) on the early immune response after experimental intramammary challenge with a heterologous killed Staphylococcus aureus strain. The effect of vaccination on milk production, clinical signs, quarter milk somatic cell count, milk polymorphonuclear neutrophilic leukocyte (PMN) concentration and viability, the concentration of antigen-specific antibodies [slime associated antigenic complex (SAAC) and J5] and their IgG1 and IgG2 subtypes in both serum and whey, and the antigen-specific IFN- $\gamma$, IL-4, and IL-17 production by blood lymphocytes after in vitro stimulation with $S$. aureus and Escherichia coli extracts were determined. A cohort of 8 clinically healthy end-term cows and heifers were conveniently selected, of which half was vaccinated with Startvac at 45 and $10 \mathrm{~d}$ before the expected calving date and half served as nonvaccinated control animals. At $15 \mathrm{~d}$ in milk, 2 contralateral quarters of each of the 8 animals were challenged with $2 \times 10^{9} \mathrm{cfu} / \mathrm{mL}$ of the formaldehyde-killed $S$. aureus C195 strain. The 2 other quarters were infused with phosphate-buffered saline and served as control quarters. The increase in both quarter milk somatic cell count and PMN concentration and the drop in milk production after $S$. aureus inoculation was less pronounced in the vaccinates than in the nonvaccinates, reflecting a less severe inflammatory response. No significant differences in PMN viability between vaccinates and nonvaccinates could be demonstrated. The serum SAAC- and J5-specific antibody concentration significantly increased across the dry period in the vaccinated animals only. The whey concentration of SAAC-specific antibodies was significantly higher in vaccinates than in nonvaccinates at both 15
\end{abstract}

Received April 4, 2016.

Accepted September 16, 2016.

${ }^{1}$ Corresponding author: Sofie.Piepers@UGent.be and $17 \mathrm{~d}$ in milk, independent from the challenge status of the quarters. No significant differences in the whey J5-specific antibody concentration were observed. Vaccination with Startvac seems to primarily evoke a Th2 response for $S$. aureus characterized by a shift toward the IgG1 antibody subtype and accompanied by a less pronounced Th1 response. The type of response against E. coli was less clear, though a weak but significant shift toward the IgG2 antibody subtype after vaccination and high IFN- $\gamma$ levels after in vitro stimulation suggest a Th1 response. The increased SAAC-specific antibody concentration in whey in vaccinates compared with nonvaccinates most probably triggers the opsonization of the inoculated $S$. aureus bacteria, resulting in a more efficient elimination of the bacteria from the mammary gland.

Key words: Escherichia coli, immune response, Staphylcoccus aureus, vaccination

\section{INTRODUCTION}

Despite a shift in the distribution of mastitis pathogens over the years from the so-called contagious bacteria toward the so-called environmental ones, Staphylococcus aureus remains a highly prevalent cause of mastitis worldwide and across many management systems (Makovec and Ruegg, 2003; Pitkälä et al., 2004; Piepers et al., 2007). Due to the potentially fast transmission from infected to uninfected animals, udder health problems on dairy farms caused by $S$. aureus are not always easy to control and a holistic approach is needed to be successful (Dufour et al., 2012). Control programs for $S$. aureus mastitis include several management procedures, such as an optimal milking routine, postmilking teat disinfection, the use of a well-functioning milking machine, segregation of known infected animals, culling of chronically infected animals, treatment of recently infected quarters, and the use of (selective) dry cow therapy, to name the most important ones (Dufour et al., 2012). 
Vaccination is another tool that can be valuable in the control of $S$. aureus mastitis, especially because the use of antimicrobials in farm animals is increasingly subjected to criticism (Kaesbohrer et al., 2012). Recently, a novel vaccine (Startvac, Laboratorios Hipra S.A., Girona, Spain) has become available for herd immunization of healthy cows and heifers. Besides the inactivated Escherichia coli (J5) strain, this vaccine contains an inactivated $S$. aureus (CP8) SP140 strain expressing the slime-associated antigenic complex (SAAC; Prenafeta et al., 2010) and an adjuvant. Although the vaccine has a label claim for reducing the incidence of subclinical mastitis and the incidence and severity of the clinical signs of mastitis caused by coliforms, S. aureus, and CNS, the immunological basis of its mechanism has yet to be discerned. Hypothetically, protection could be the result of an increased opsonization of bacteria via increased antibody concentrations in blood and milk combined with a more efficient phagocytosis and killing of bacteria by PMN. Enhancement of the PMN viability and activity could be another potential mechanism. Although both hypotheses are plausible, none of them has yet been investigated in depth.

The main objective of our study was to conduct an experimental trial exploring the effect of vaccination with Startvac on the early immune response after experimental intramammary challenge with a killed heterologous $S$. aureus strain. Using killed rather than alive bacteria allowed for a more straightforward interpretation of the data, as all cows are then exposed to an equal burden of bacteria throughout the whole trial. Milk production, clinical signs, quarter milk SCC (qSCC), milk PMN concentration and viability, and both the concentration of antigen-specific antibodies (SAAC and J5) and their IgG1 and IgG2 subtypes in both serum and whey were determined. In addition, the antigen-specific IFN- $\gamma$, IL-4, and IL-17 production by blood lymphocytes after in vitro stimulation with $S$. aureus and E. coli extracts was determined. Also, several clinical parameters were observed in vaccinated and nonvaccinated cows.

\section{MATERIALS AND METHODS}

The study was approved by the ethical committee of the Faculty of Veterinary Medicine, Ghent University (EC 2010/114).

\section{Study Design}

Table 1 presents a schematic overview of the study design and the different data and samples collected from the 4 vaccinated and 4 nonvaccinated animals that were included in the data analysis.
Animal Selection. In total, 4 Holstein nonlactating heifers and 4 Holstein cows were included in the intramammary challenge study. The number of animals needed for our study was determined by a sample size calculation performed in WinEpiscope 2.0 (Thrusfield et al., 2001) with a power of $80 \%$ and a confidence level of $95 \%$ for the outcome variables of main interest, including the milk PMN viability (Mehrzad et al., 2004; Piepers et al., 2009a,b), the concentration of antigenspecific antibodies against $S$. aureus, and the IgG1 and IgG2 subtypes in both serum and whey (Luby et al., 2007; Prenafeta et al., 2010); input parameters (i.e., expected biological relevant difference and SD) were derived from literature.

For that reason, a cohort of 8 clinically healthy endterm cows and 8 end-term heifers were conveniently selected (i.e., based on the expected calving date) at the research dairy farm of the Faculty of Veterinary Medicine, Ghent University, Belgium (Biocentrum Agri-Vet, Melle, Belgium). Half of these animals were vaccinated intramuscularly at 45 and $10 \mathrm{~d}$ before the expected calving date with Startvac (Laboratorios Hipra S.A.) containing the inactivated E. coli J5 strain, the $S$. aureus SP140 strain (capsular polysaccharide type 8 strain) expressing SAAC (Prenafeta et al., 2010), and liquid paraffin adjuvant. Nonvaccinated animals did not receive any sham vaccination. After calving, duplicate milk samples $(5 \mathrm{~mL})$ were aseptically collected for bacteriological culturing and determination of the qSCC twice, between 2 and 6 DIM and again between 10 and 14 DIM after the first streams of milk were discarded. Only cows and heifers in which all quarters were culture-negative in at least 1 of the duplicate samples at both samplings and had a qSCC $\leq 200,000$ cells $/ \mathrm{mL}$ at both samplings were eligible for inclusion. Animals for which 1 or more quarters did not fulfil these conditions or animals with general signs of disease or with signs of clinical mastitis [any abnormal aspect of the milk (flakes, clots, and a watery or other unusual appearance), in combination or not with other visible abnormalities of the udder (redness, swelling, and so on)] between the moment of first selection and the start of the actual trial at 15 DIM were excluded. The first 2 vaccinated heifers and 2 vaccinated cows and the first 2 nonvaccinated heifers and 2 nonvaccinated cows that fulfilled all conditions were selected for the actual trial and subject to intramammary challenge at 15 DIM.

Preparation of Bacteria. After the formaldehyde inactivation, bacteria were washed 3 times with PBS. In each wash, cells were harvested by centrifugation $(3,000$ $\times g$ for $10 \mathrm{~min}$ at $4^{\circ} \mathrm{C}$ ) and the pellet was resuspended with PBS. The endotoxin content of the PBS was $<1$ endotoxin units $/ \mathrm{mL}$, determined by the chromogenic 
VACCINATION AGAINST MASTITIS IN LACTATING COWS
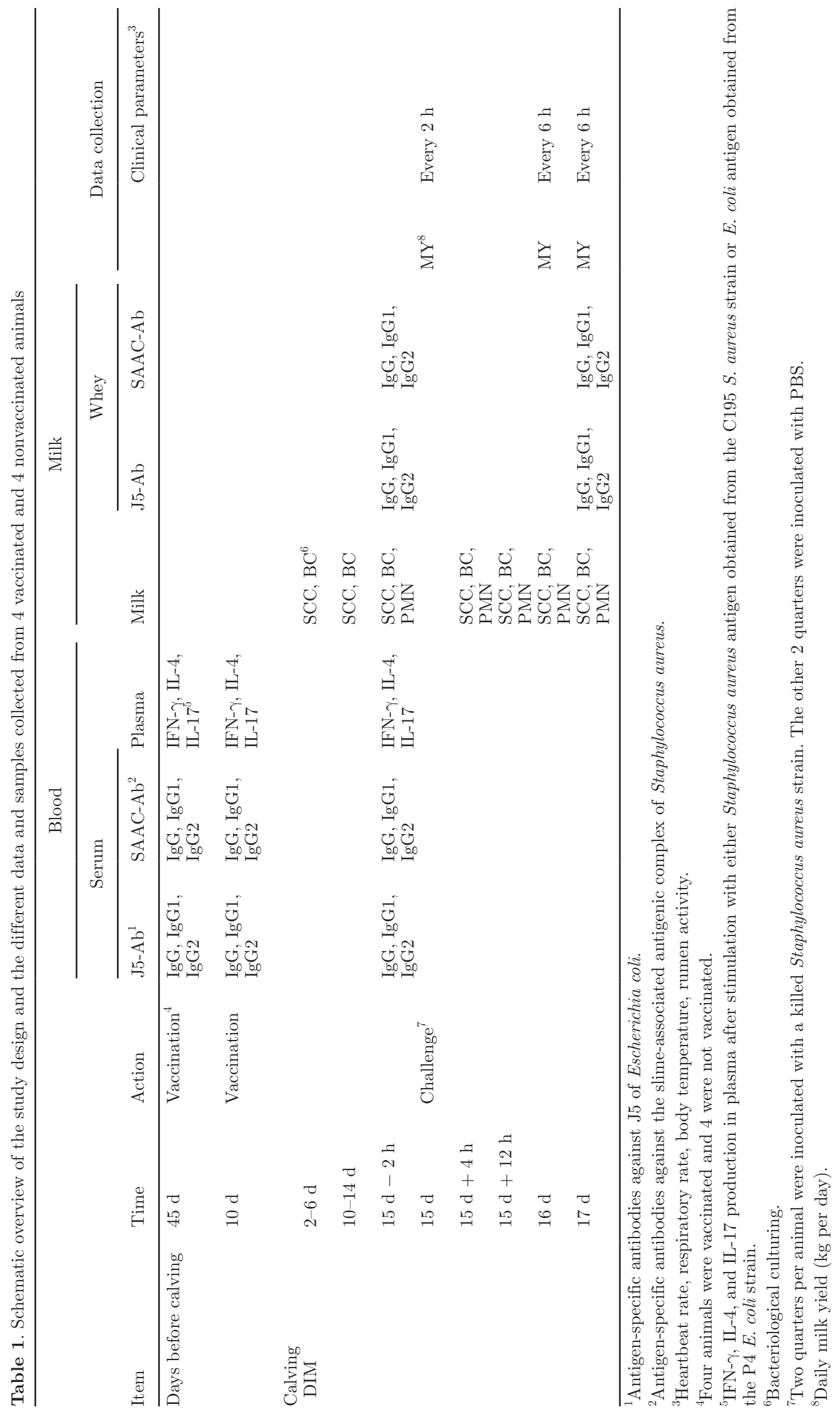

Journal of Dairy Science Vol. 100 No. 1, 2017 
limulus amebocyte lysate method (Endochrome KTM, Charles River, Barcelona, Spain).

Intramammary Challenge. At 15 DIM, 2 contralateral quarters of each of the 8 animals were challenged with $2 \times 10^{9} \mathrm{cfu} / \mathrm{mL}$ of the formaldehyde-killed $S$. aureus $\mathrm{C} 195$ strain (Laboratorios HIPRA S.A.) $2 \mathrm{~h}$ after morning milking using a sterile human bladder catheter (Vygon, Ecouen, France). The 2 other quarters were infused with PBS and served as control quarters. In 2 heifers and 2 cows the left front and right hind quarter were challenged, whereas in the other 4 animals the right front and left hind quarter were challenged. The inoculation protocol included cleaning of udder and teats with an individual dry paper cloth, cleaning of the teats with a premilking disinfection solution (Oxyfoam, Ecolab, Groot-Bijgaarden, Belgium), wiping the teats with an individual dry paper, disinfecting the teats with $70 \%$ alcohol-saturated cotton plugs, infusion of the control quarters with $5 \mathrm{~mL}$ of sterile PBS, infusion of the 2 other quarters with $5 \mathrm{~mL}$ of the bacterial inoculum, gentle massage of all quarters for $30 \mathrm{~s}$, and disinfection of each teat with a postmilking teat disinfection solution (Io-Shield, Ecolab).

\section{Sample and Data Collection}

Blood Samples. Blood samples were collected from the tail vein using Vacutainer tubes containing a clot activator (Terumo, Leuven, Belgium) at 45 and $10 \mathrm{~d}$ before the expected calving date and at $2 \mathrm{~h}$ before inoculation at 15 DIM to determine the SAAC- and J5specific serum antibody concentrations. An additional tube of heparinized blood (10 mL; Terumo, Leuven, Belgium) was collected from the tail vein to determine the in vitro IFN- $\gamma$, IL-4, and IL-17 production in whole blood after $S$. aureus and $E$. coli challenge each time point (3 times).

Quarter Milk Samples. Besides the duplicate quarter milk samples that were collected between 2 and 6 DIM and between 10 and 14 DIM as part of the selection process, duplicate quarter milk samples $(5 \mathrm{~mL})$ were aseptically collected for bacteriological culturing and determination of qSCC at $2 \mathrm{~h}$ before challenge and at different time points after challenge (Table 1). Bacteriological culturing was performed to detect new naturally occurring IMI. A quarter was considered as infected if the pathogen isolated from the duplicate milk samples was phenotypically identical. When the duplicate milk samples were contaminated, the data for that quarter were considered missing for that specific time point only. Isolation of a mastitis pathogen from only 1 of the duplicate milk samples was considered nonsignificant growth and eventually considered as culture-negative. Additionally, quarter milk samples were collected for the quantification of PMN concentration and viability at different time points between 15 and 17 DIM (200 mL) and to determine the SAAC- and J5-specific quarter milk antibody concentration at 15 and 17 DIM (Table 1).

Clinical Parameters. Several clinical parameters (heartbeat rate, respiratory rate, body temperature, and rumen activity) were recorded every $2 \mathrm{~h}$ at 15 DIM and every $6 \mathrm{~h}$ at 16 and 17 DIM (Table 1). Daily milk yield (MY) was determined every day at 15 and 17 DIM.

\section{Laboratory Analyses}

Bacterial Identification. Bacteriological culturing was done as previously described (Piepers et al., 2007) and performed at the laboratory of the Mastitis and Milk Quality Research Unit (Merelbeke, Belgium). Also, from each milk sample obtained from quarters inoculated with the killed $S$. aureus strain, DNA was extracted and a multiplex real-time PCR (PathoProof, Thermo Fisher Scientific Inc., Pittsburgh, PA) was performed to verify whether $S$. aureus was detectable. Cycle threshold values above 37 were considered as negative, values between 12 and 37 were considered as PCR-positive.

$\boldsymbol{q S C C}$. Quarter milk SCC was quantified by electronic counting (Direct Cell Counter, DeLaval, Gent, Belgium).

Milk PMN Viability and Concentration. The milk used to isolate PMN was divided into several 50-mL Falcon tubes (BD Biosciences, Erembodegem, Belgium) and diluted 1:1 with PBS. All tubes were centrifuged $(600 \times g)$ for $15 \mathrm{~min}$ at $4^{\circ} \mathrm{C}$, the cream layer and supernatant were removed, and each pellet was suspended into $10 \mathrm{~mL}$ of PBS. Two pellets were merged together and centrifuged again $\left(200 \times g, 4^{\circ} \mathrm{C}\right)$ for $10 \mathrm{~min}$; this was repeated 2 more times. Subsequently, milk PMN were differentiated from other milk cells by a 2-step fluorescent immunolabeling using a primary anti-bovine monoclonal granulocyte antibody (CH138A; VMRD Inc., Pullman, WA) and an Alexa 647-labeled goat anti-mouse IgM secondary antibody (Molecular Probes, Invitrogen, Waltham, MA) as previously described (Piepers et al., 2009a). To identify apoptotic and necrotic PMN, a double fluorescein isothiocyanate (FITC)-annexin-V (Roche, Indianapolis, IN) and propidium iodide (Sigma-Aldrich, Bornem, Belgium) staining was used. The PMN positive for FITC and negative for propidium iodide were considered as (early) apoptotic, whereas PMN that were positive for both FITC and PI were considered necrotic. Polymorphonuclear neutrophilic leukocytes that were negative for both stains were considered viable (Van 
Oostveldt et al., 2001; Piepers et al., 2009a). Milk PMN viability was expressed as the proportion of viable milk PMN.

SAAC- and J5-Specific Antibody Titers in Serum and Whey. The concentration of the SAACspecific antibodies in serum and whey was determined as previously described (Prenafeta et al., 2010). Serum samples were assayed in duplicate for IgG SAAC-specific antibodies using an SAAC-based indirect ELISA. Briefly, 96-well plates were coated with purified SAAC and blocked with a BSA solution. Wells were incubated with diluted bovine serum samples (1:100), and protein G conjugated with peroxidase (Pierce, Thermo Fisher Scientific Inc.) was used to detect the bound total IgG antibodies. For the IgG1 and IgG2 antibody titers, a sheep anti-bovine IgG1 and a sheep anti-bovine IgG2 conjugated with peroxidase were used instead of protein G. Finally, wells were incubated with a chromogenic substrate for the peroxidase and the absorbance of the reaction was measured as optical density (OD) using a microplate reader at $405 \mathrm{~nm}$. The SAAC-specific antibodies in whey from each quarter were detected by the SAAC based on the indirect ELISA procedure using nondiluted whey samples. Whey was prepared by centrifugation $\left(20,000 \times g\right.$ for $30 \mathrm{~min}$ at $\left.4^{\circ} \mathrm{C}\right)$. The mean values of the OD in the total IgG determinations were converted into a relative index percent (RIPC) using the formula

$$
\text { RIPC }=(\text { OD sample }- \text { OD negative control }) /
$$

(OD positive control - OD negative control) $\times 100$.

Bovine sera without SAAC-specific antibodies and with high antibody titers against the SAAC antigen were used as negative and positive control samples, respectively. A similar method was applied for the determination of the J5-specific antibodies in serum and whey, but using 96-well plates coated with J5 antigen instead of the SAAC antigen. The J5 antigen used in the ELISA was the same strain as in the Startvac vaccine itself. A suspension of $E$. coli J5 cells in PBS was heat inactivated and diluted with carbonate buffer 100 $\mathrm{m} M, \mathrm{pH} 9.6$, to coat the 96-well plates. After incubation for 18 to $24 \mathrm{~h}$ at 2 to $8^{\circ} \mathrm{C}$, the plates were blocked with Stabilcoat (SurModics Inc., Eden Prairie, MN). Bovine sera without E. coli J5-specific antibodies and with high antibody titers against the $E$. coli J5 antigen were used as negative and positive control samples, respectively.

Antigen-Specific IFN- $\gamma, I L-4$, and IL-17 Production. Within $2 \mathrm{~h}$ after collection, $0.9 \mathrm{~mL}$ of each heparinized blood sample was added in 4 wells of a 24 flat-well tissue culture plate (Nunc, Cat. No. 142475,
Thermo Fisher Scientific Inc.). Then control and test samples were added to the appropriate wells. One well received $0.1 \mathrm{~mL}$ of PBS (unstimulated control), 1 well received $0.1 \mathrm{~mL}$ of Concanavalin A (Sigma-Aldrich) at a working solution of $100 \mu \mathrm{g} / \mathrm{mL}$ (positive control to ensure the ability of the cells to respond to a stimulation), 1 well received $0.1 \mathrm{~mL}$ of $S$. aureus antigen obtained from the C195 $S$. aureus strain, and 1 well received $0.1 \mathrm{~mL}$ of E. coli antigen obtained from the P4 E. coli strain. The $S$. aureus and E. coli antigens consisted of killed and sonicated cells. Briefly, bacterial cells were inactivated with formaldehyde and washed 3 times in PBS, then the suspension was sonicated for $105 \mathrm{~s}$ at 130 W (Labsonic U, Standard Probe 5T, B. Braun, Barcelona, Spain). Finally, the total protein concentration of the antigens was determined by the bicinchoninic acid method (Pierce BCA Protein Assay Kit, Thermo Fisher Scientific Inc.) and adjusted to $1 \mathrm{mg} / \mathrm{mL}$. Wells containing whole blood samples were stimulated with $0.1 \mathrm{mg}$ of each antigen $(0.1 \mathrm{~mL} /$ well $)$. Once all samples were delivered to the appropriate wells, the plate was incubated at $37^{\circ} \mathrm{C}$ with $5 \% \mathrm{CO}_{2}$ for $24 \mathrm{~h}$. The plate was then centrifuged at $500 \times g$ at $4^{\circ} \mathrm{C}$ for $10 \mathrm{~min}$. After centrifugation, the supernatants were collected and stored at $-20^{\circ} \mathrm{C}$. The IFN- $\gamma$ in the supernatant was detected using a commercial ELISA-test kit (Kit ELISA Bovigam TB, Thermo Fisher Scientific Inc.). The IL-4 and IL-17 production in the supernatant were detected using commercial ELISA-test kits (Kit ELISA Bovine IL-4 Screening Set, Thermo Fisher Scientific Inc.; Kit ELISA Bovine IL-17 VetSet, Kingfisher Biotech Inc., Saint Paul, MN). Similar to the total IgG determinations in serum and whey, the mean values of the OD were converted into RIPC. Finally, the RIPC of the unstimulated control was subtracted from the RIPC of the test sample.

\section{Statistical Analyses}

Quarter-Level Analyses. Linear mixed regression models including animal (RANDOM statement) and quarter within an animal (REPEATED statement) as random effects (PROC MIXED, SAS 9.3, SAS Institute Inc., Cary, NC) were fit to evaluate the association between the animals' vaccination status before calving and the evolution of qSCC, milk PMN concentration, and milk PMN viability over time. A natural logarithmic transformation of $\mathrm{qSCC}$ and a $\log _{10}$ transformation the milk PMN concentration were performed to obtain a normal distribution. All models included the animal vaccination status (vaccinated versus nonvaccinated), the quarter challenge status (infused with PBS versus inoculated with the killed $S$. aureus strain), the presence 
of new IMI (infected versus noninfected, categorical), and the time of sampling (5 time points, categorical) as well as all 1- and 2-way interaction terms between the animal vaccination status, the quarter challenge status, and the time of sampling. Naturally occurring IMI were included as a binary predictor variable at the time of occurrence and indicated as 1, modeling the potential confounding effect of naturally occurring IMI on the different outcome variables. Quarters without new naturally occurring IMI were coded as zero at every time point. Similar models only including 2 repeated measurements per quarter were fit to evaluate the association between the animal vaccination status and the quarter milk SAAC- and J5-specific IgG, IgG1, and IgG2 antibody concentration at 15 and 17 DIM. In all models, all 1-way interaction terms (animal vaccination status $\times$ quarter challenge status; animal vaccination status $\times$ time of sampling; quarter challenge status $\times$ time of sampling) and the 2-way interaction term between animal vaccination status, quarter challenge status, and time of sampling were included.

To determine the association between the PCR result at $48 \mathrm{~h}$ postchallenge (PC) and the cow's vaccination status, a Fisher's Exact Test was performed (SPSS 22.0, SPSS Inc., Chicago, IL). The odds ratio and $95 \%$ confidence interval were calculated.

Cow-Level Analyses. Linear mixed regression models adjusting for clustering of repeated measurements within cows (REPEATED statement) were fit to evaluate the association between the animal vaccination status before calving and the serum SAAC- and J5-specific IgG, IgG1, and IgG2 antibody concentration, as well as the antigen-specific IFN $\gamma$, IL-4, and IL-17 production by blood lymphocytes after in vitro stimulation with $S$. aureus and $E$. coli extracts at 45 and $10 \mathrm{~d}$ before the expected calving date and at 15 DIM before inoculation (PROC MIXED, SAS 9.3, SAS Institute Inc.), respectively. The model included the animal vaccination status (vaccinated versus nonvaccinated, categorical), the time of sampling (3 time points, categorical), and the 1-way interaction term between both variables. A similar model including 3 repeated measurements per animal was fit to evaluate the association between the animals' vaccination status and the daily MY at 15, 16, and 17 DIM.

Overall. In all linear mixed models, a compound symmetry correlation structure was used to account for the clustering of repeated measurements within a quarter or animal. For all linear mixed models, the goodness-of-fit measures included $-2 \times \log$ likelihood, the Akaike information criterion, and the Bayesian information criterion. Residuals were evaluated graphically and were graphed against the predicted values.
Significance was assessed at $P<0.05$. A Bonferroni correction was applied to correct for multiple comparisons.

\section{RESULTS}

\section{Descriptive Statistics}

All animals remained clinical healthy during the trial period. Challenge did not affect clinical parameters such as heartbeat rate, respiration rate, manure consistency, or appetite. The average body temperature 2 $\mathrm{h}$ before inoculation was $38.8^{\circ} \mathrm{C}$ (range $=37.7-39.1^{\circ} \mathrm{C}$ ) and $38.6^{\circ} \mathrm{C}$ (range $=38.0-39.1^{\circ} \mathrm{C}$ ) for the vaccinated and nonvaccinated animals, respectively. In both groups, body temperature slightly increased from 38.4 and $38.6^{\circ} \mathrm{C}$ at the time of challenge for the vaccinated and nonvaccinated animals, respectively, to 39.3 and $39.9^{\circ} \mathrm{C}$, respectively, at $33 \mathrm{~h} \mathrm{PC}$, and decreased to 39.0 and $39.2^{\circ} \mathrm{C}$, respectively, at $48 \mathrm{~h} \mathrm{PC}$.

Four hours PC, a positive PCR result was obtained in 7 of the 8 quarters inoculated with the killed $S$. aureus strain from nonvaccinated animals and in all 8 quarters inoculated with the killed $S$. aureus from vaccinated animals. Twelve hours PC, all quarters inoculated with the killed $S$. aureus strain were PCR-positive for S. aureus. Forty-eight hours PC, 7 quarters from the nonvaccinated animals and 2 quarters from the vaccinated animals were still PCR-positive. Quarters from vaccinated animals had 0.048 odds of still being PCRpositive at 17 DIM compared with quarters of nonvaccinated animals $(P=0.04 ; 95 \% \mathrm{CI}=0.003-0.665)$.

In 4 control quarters and $3 S$. aureus-challenged quarters from 2 vaccinated and 3 nonvaccinated animals, a new naturally occurring IMI was observed at 1 or more samplings (13 positive samplings of 160). Three of those IMI were caused by Corynebacterium bovis and 10 were caused by CNS.

\section{Daily MY and Cellular Immune Response}

The average daily MY per animal was $33.2 \mathrm{~kg}$ (range $=19.0-46.0 \mathrm{~kg}$ ) at the onset of the trial. In the nonvaccinated group average daily MY tended to decrease from $34.2 \mathrm{~kg} / \mathrm{d}$ at 15 DIM to $30.5 \mathrm{~kg} / \mathrm{d}$ at 16 DIM $(P$ $=0.06)$. In the vaccinated group, no significant differences in average daily MY were observed over time. In this group, the average daily MY even increased from $33.7 \mathrm{~kg} / \mathrm{d}$ at 15 DIM to $36.5 \mathrm{~kg} / \mathrm{d}$ at 16 DIM $(P=$ 0.16). In both groups of animals, the qSCC of the $S$. aureus-challenged quarters increased over time $(P<$ 0.001). The qSCC in the $S$. aureus-challenged quarters was higher than in the control quarters in the nonvacci- 


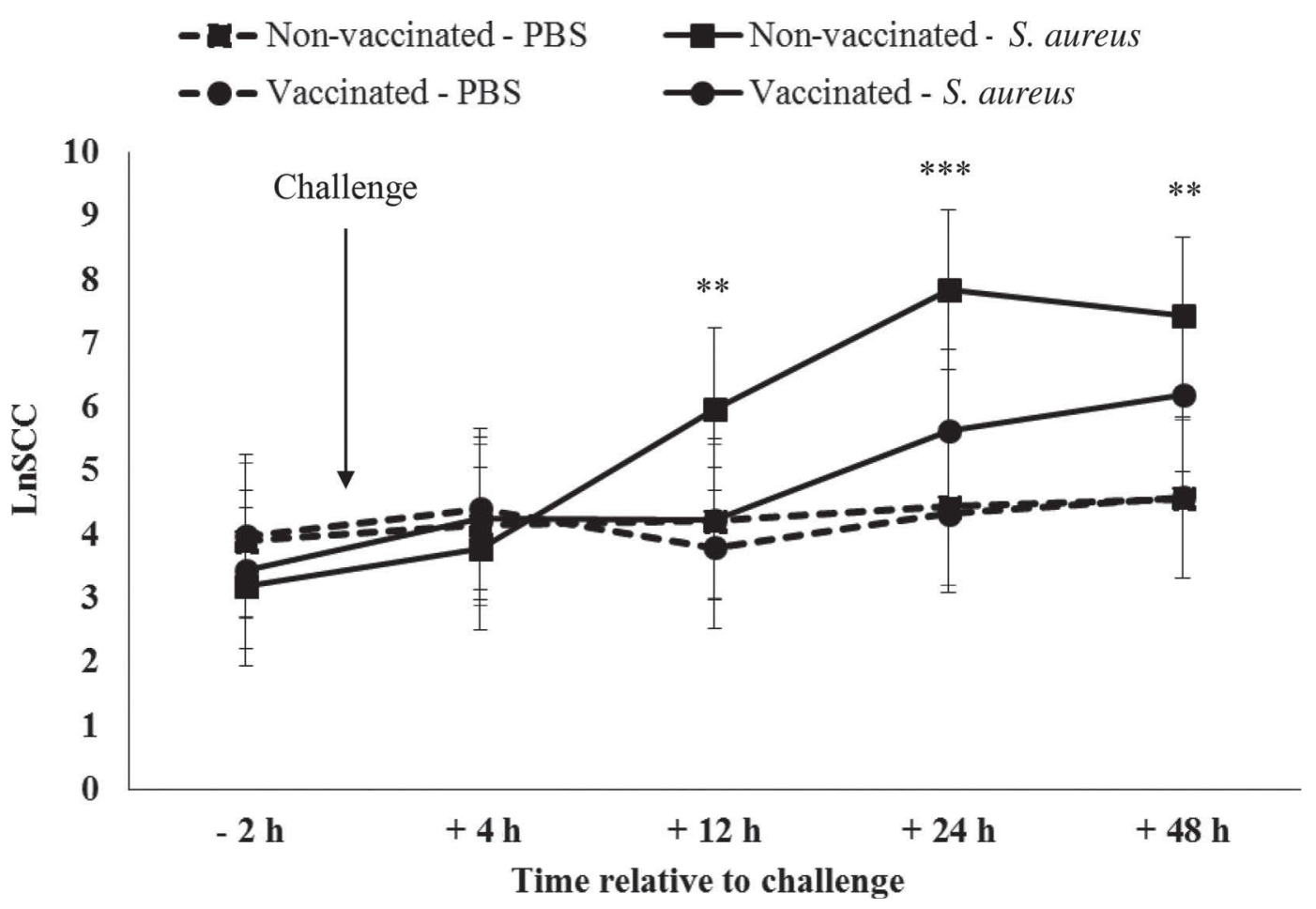

Figure 1. Evolution of the natural log-transformed quarter milk SCC (LnSCC \pm SE) for quarters infused with PBS (dashed line) and

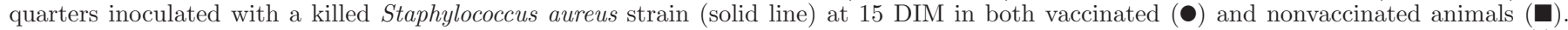
Significant differences between the control quarters and $S$. aureus-challenged quarters in the nonvaccinated animals only are indicated by $* * P$ $<0.01 ; * * * P<0.001$.

nated animals only $(P<0.001)$, although the difference depended on the time of sampling (Figure 1). In the vaccinated group, the qSCC in the $S$. aureus-challenged quarters was not significantly different from the qSCC in the control quarters $(P=0.22$; Figure 1$)$. Similar results were obtained for the milk PMN concentration (Figure 2); quarters challenged with the killed $S$. aureus strain had a significantly higher PMN concentration than the control quarters from $24 \mathrm{~h} \mathrm{PC}$ and on (Figure 2). Still, the difference in PMN viability between the S. aureus-challenged quarters and control quarters during the trial period did not depend on the vaccination status of the animal $(P=0.99$; Figure 3$)$.

\section{SAAC- and J5-Specific IgG1, IgG2, and Total Antibodies in Serum}

The serum concentration of both SAAC- and J5specific antibodies increased between $45 \mathrm{~d}$ before the expected calving date and 15 DIM in the vaccinated animals only $(P<0.001$ and $P=0.002$, respectively). Vaccinated animals had significantly higher SAAC- and J5-specific antibody serum concentrations at 15 DIM (i.e., prechallenge) than the nonvaccinated animals $(P$
$<0.001$ and $P=0.04$, respectively; Figures $4 \mathrm{~A}$ and $5 \mathrm{~A})$. The difference in increase between $45 \mathrm{~d}$ before the expected calving date and 15 DIM was clearly more pronounced for the serum SAAC-specific antibodies $(P$ $<0.001)$ than for the serum J5-specific antibodies $(P$ $=0.051$ ). When analyzing the IgG subtypes (Table 2 ), the serum SAAC-specific IgG1 concentration increased from dry off to 15 DIM $(P<0.001)$ in the vaccinated animals only. Likewise, the OD for the serum SAACspecific IgG1 at 15 DIM was higher in the serum of the vaccinated animals than in the serum of the nonvaccinated animals $(P=0.005)$. The OD for the serum SAAC-specific IgG2 did not significantly increase between $45 \mathrm{~d}$ before the expected calving date and 15 DIM (Table 2; $P=1.00$ ). The OD for serum J5-specific IgG1 tended to significantly increase between dry off and 15 DIM in the vaccinated animals $(P=0.08)$, whereas the serum J5-specific IgG2 concentration increased between those 2 sampling points $(P=0.02)$. The OD for the serum J5-specific IgG1 and IgG2 did not significantly differ between vaccinated and nonvaccinated animals at dry off $(P=1.00), 10 \mathrm{~d}$ before the expected calving date $(P=1.00)$, or 15 DIM $(P=1.00$ and $P=0.74$, respectively). 


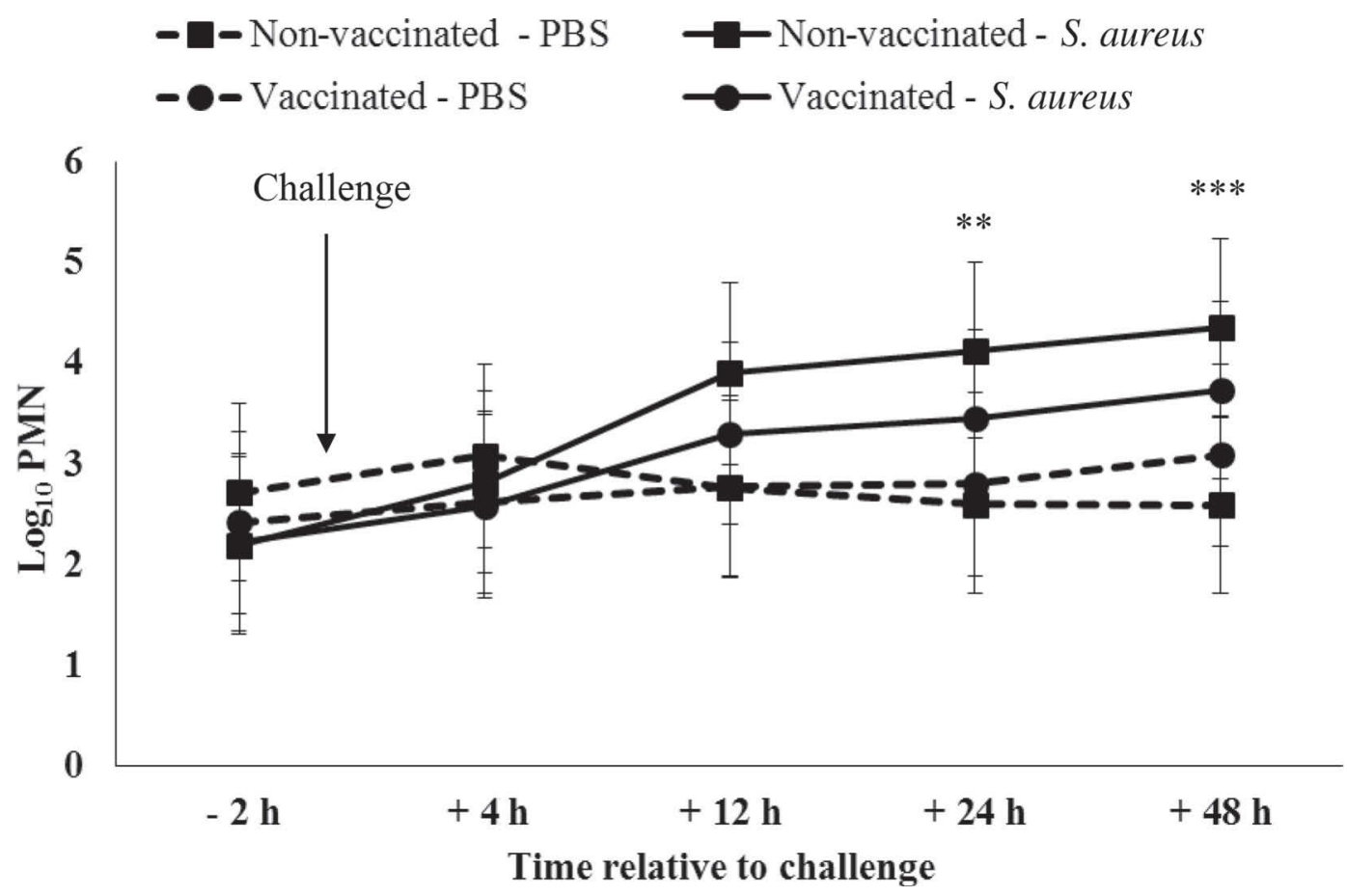

Figure 2. Evolution of the $\log _{10}$-transformed quarter milk PMN concentration $\left(\log _{10} \mathrm{PMN} \pm \mathrm{SE}\right.$ ) for quarters infused with PBS (dashed line) and quarters inoculated with a killed Staphylococcus aureus strain (solid line) at 15 DIM in both vaccinated (-) and nonvaccinated animals (ם). Significant differences between the control quarters and $S$. aureus-challenged quarters in the nonvaccinated animals only are indicated by $* * P<0.01 ; * * * P<0.001$.

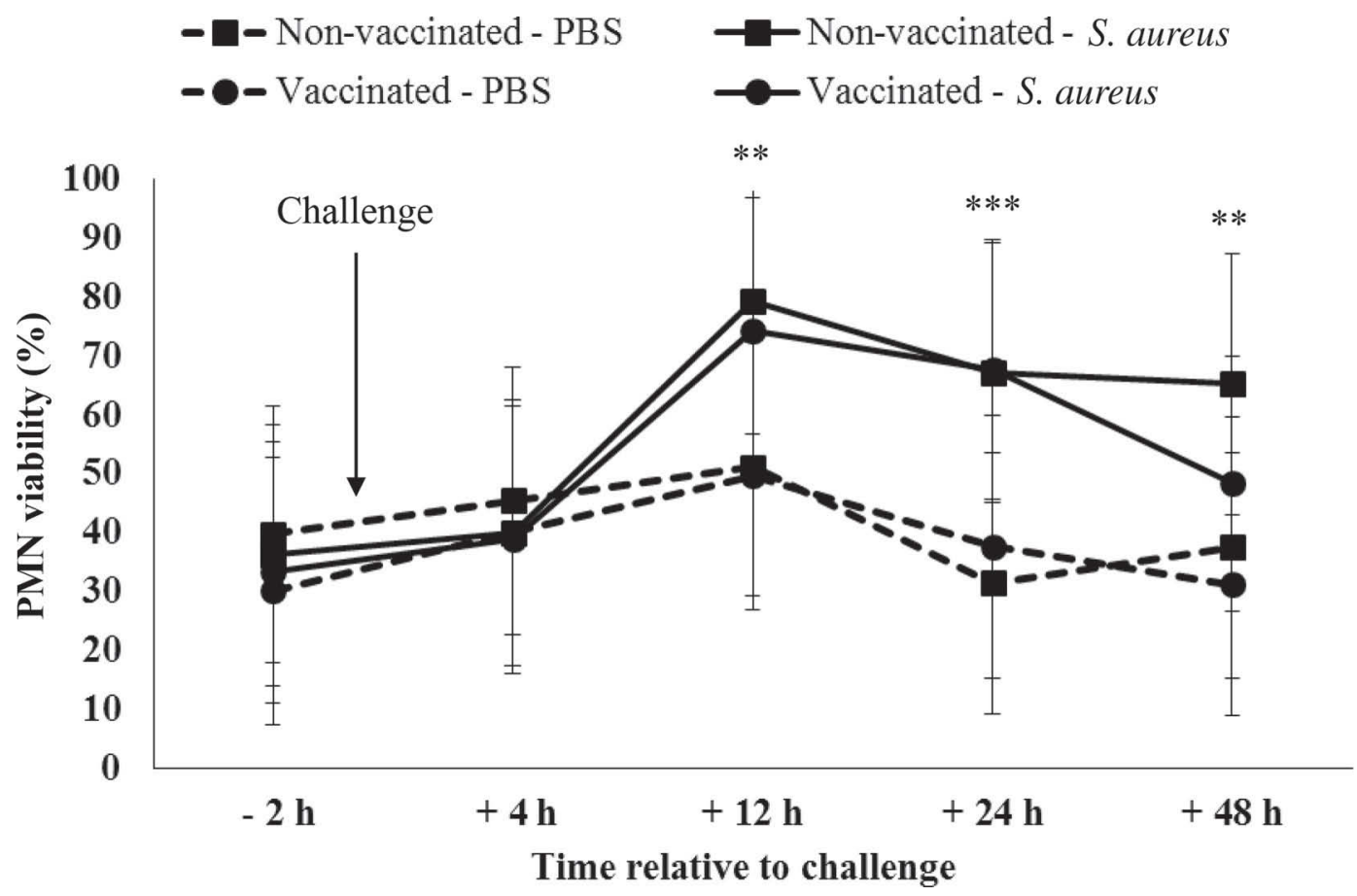

Figure 3. Evolution of the quarter milk PMN viability $(\% \pm \mathrm{SE}$ ) for quarters infused with PBS (dashed line) and quarters inoculated with a killed Staphylococcus aureus strain (solid line) at 15 DIM in both vaccinated (-) and nonvaccinated animals (ם). Significant differences between the control quarters and $S$. aureus-challenged quarters independently from the vaccination status of the animal: ${ }^{* *} P<0.01 ;{ }^{* * *} P<0.001$. 

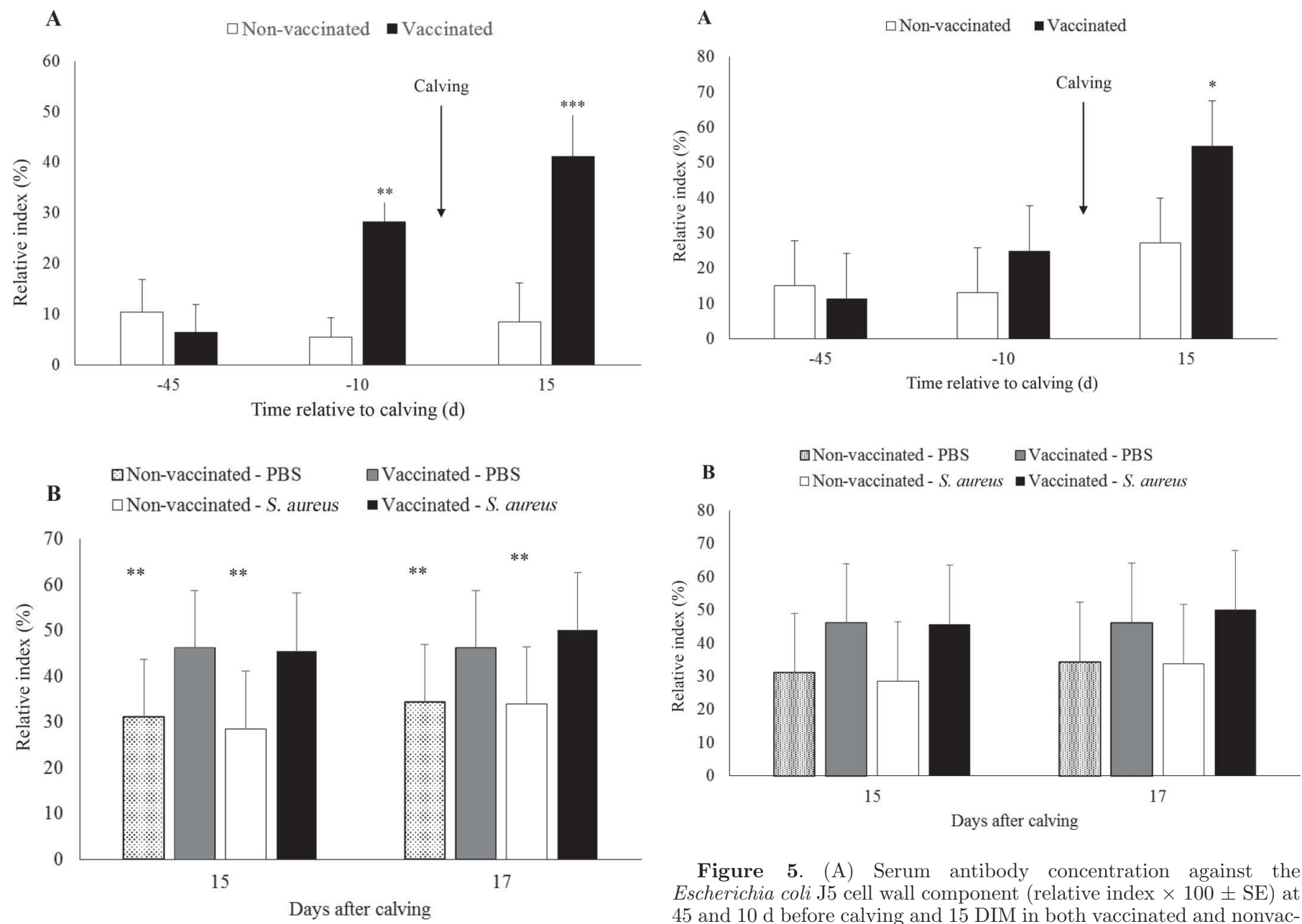

Figure 5. (A) Serum antibody concentration against the Escherichia coli J5 cell wall component (relative index $\times 100 \pm \mathrm{SE}$ ) at 45 and $10 \mathrm{~d}$ before calving and 15 DIM in both vaccinated and nonvaccinated animals and (B) the whey antibody concentration against the Escherichia coli J5 cell wall component (relative index $\times 100 \pm \mathrm{SE}$ ) for quarters infused with PBS and quarters inoculated with a killed Staphylococcus aureus strain at 15 and 17 DIM in both vaccinated and nonvaccinated animals. Significant differences between vaccinated and nonvaccinated animals independently from the inoculation status of the quarters (only for whey) were indicated by $* P<0.05$. quarters inoculated with a killed Staphylococcus aureus strain at 15 and 17 DIM in both vaccinated and nonvaccinated animals. Significant differences between vaccinated and nonvaccinated animals independently from the inoculation status of the quarters (only for whey) were indicated by ${ }^{* *} P<0.01 ;{ }^{* * *} P<0.001$.

\section{SAAC- and J5-Specific IgG1, IgG2, and Total Antibodies in Whey}

The whey concentration of SAAC-specific antibodies was significantly higher in vaccinated animals than in nonvaccinated animals, independent from the inoculation status of the quarters and the time of sampling $(P$ $=0.002 ;$ Figures $4 \mathrm{~B}$ and $5 \mathrm{~B})$. The whey concentration of J5-specific antibodies observed in vaccinated animals was not significantly different at 15 or at 17 DIM from the ones in the nonvaccinated animals $(P=0.34$ and $P$ $=0.52$, respectively). When analyzing the IgG isotypes (Table 2), the OD for the whey SAAC-specific IgG1

was significantly higher in the whey of the vaccinated animals than in the whey of the nonvaccinated animals PC $(P=0.01)$, although the difference was more pronounced at 15 than at 17 DIM (Table 2). In the vaccinated animals only, the whey SAAC-specific IgG1 OD values significantly decreased between 15 and 17 DIM $(P=0.005)$ (Table 2). No significant differences were observed in the whey SAAC-specific IgG2 OD values between 15 and 17 DIM in vaccinates $(P=1.00)$. In the nonvaccinated animals, the whey SAAC-specific IgG2 concentration was significantly higher at 17 than at 15 DIM $(P=0.01)$. The OD for the whey J5-specific IgG1 and IgG2 did not significantly differ between vaccinated and nonvaccinated animals at 15 DIM (prechallenge) or at 17 DIM $(P=1.00)$. However, in the vaccinated 
PIEPERS ET AL.

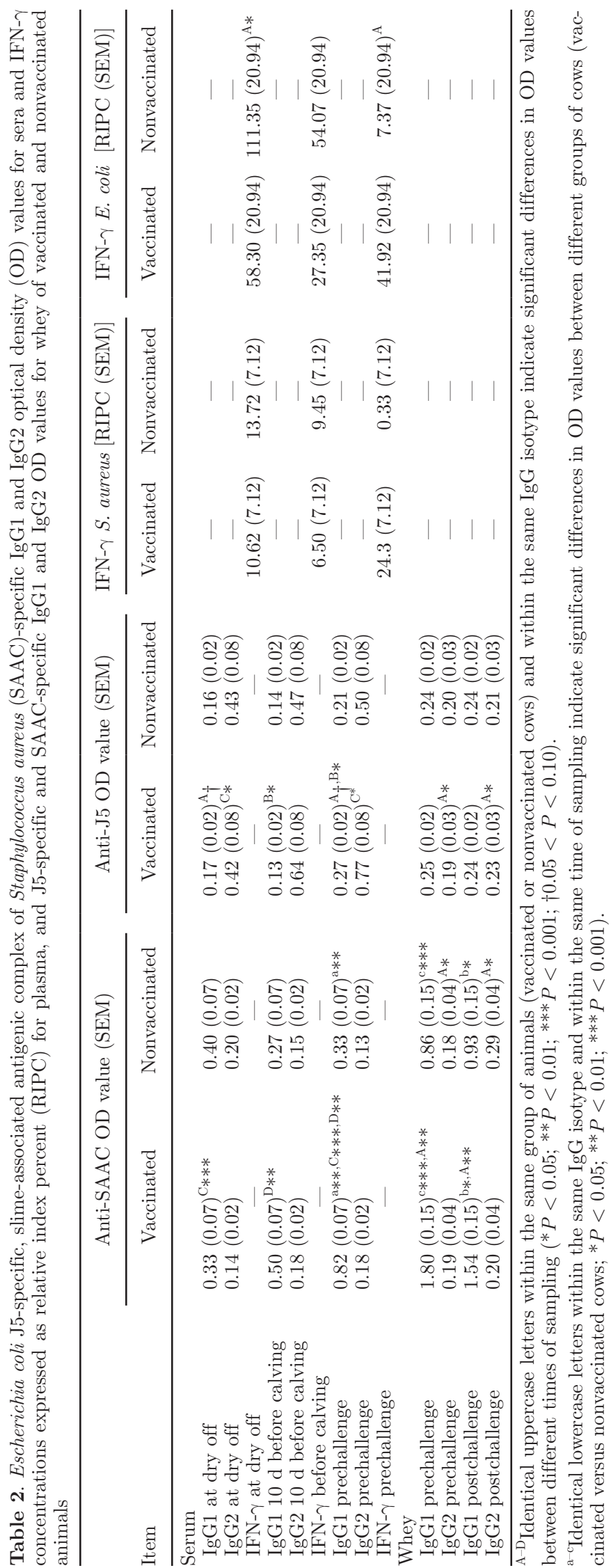


animals only the whey J5-specific IgG2 concentration significantly increased from 15 to 17 DIM $(P=0.03)$.

\section{IFN-y, IL-4, and IL-17 Production After Stimulation with $S$. aureus and $E$. coli}

No statistically significant changes in the serum IFN- $\gamma$ RIPC values were observed over time neither after $S$. aureus nor after E. coli stimulation (Table 2). Still, the IFN- $\gamma$ production by the blood lymphocytes before and after in vitro stimulation with $S$. aureus and E. coli strongly varied among the animals. Four animals (2 vaccinated and 2 nonvaccinated animals) produced already high levels of IFN- $\gamma$ after in vitro stimulation with $S$. aureus at $45 \mathrm{~d}$ before calving (i.e., before vaccination). Excluding those animals from the analyses (i.e., 2 vaccinated and 2 nonvaccinated animals) resulted in a significantly higher IFN- $\gamma$ production, expressed as RIPC, in the vaccinated animals compared with their nonvaccinated counterparts (19.2 vs. $4.9 ; P=0.03)$. The difference in IFN- $\gamma$ was most pronounced at 15 DIM (44.15 vs. $-0.35 ; P=0.02$ ). Similar observations to those obtained with $S$. aureus were found after in vitro stimulation with $E$. coli. Differences between vaccinated and nonvaccinated animals could not be statistically determined, as only 1 nonvaccinated animal showed low levels of IFN- $\gamma$ at 45 $\mathrm{d}$ before calving (i.e., before vaccination) after in vitro stimulation with E. coli. Still, the IFN- $\gamma$ production in the latter animal after in vitro stimulation with $E$. coli did not follow the same increasing trend as described above for its vaccinated counterparts. Neither IL-4 nor IL-17 could be detected in the supernatants at any sampling point neither after in vitro stimulation with $S$. aureus or with E. coli.

\section{DISCUSSION}

Our study investigated the potential mechanisms behind the recently described protective effects of the novel mastitis vaccine Startvac against $S$. aureus and E. coli (Schukken et al., 2014; Bradley et al., 2015). Vaccination of dairy cows with Startvac was associated with a less severe inflammatory response derived from the less pronounced PMN influx PC in the vaccinated animals compared with the nonvaccinated animals. Also, the difference in inflammatory response between both groups of animals appeared to be reflected in the substantial drop in milk production in the first days PC in the nonvaccinated animals only. Immunization with the vaccine appears to confer protection at least partly by increasing antibodies against SAAC and J5. Vaccinates had more SAAC- and J5-specific antibodies at 15 DIM both in sera and whey than the nonvacci- nates, with a class switch to mainly IgG1 for the SAAC antibodies.

The severity of the inflammatory reaction in case of IMI is to a large extent determined by the host immune responses and to a lesser extent by the bacterial characteristics (Burvenich et al., 2003; Piccart et al., 2016). To facilitate the between-cow comparison of a different vaccination status, we decided to use killed rather than alive bacteria, particularly because the number of animals that was included was limited. For killed bacteria, no bacterial growth will take place and, thus, all cows are exposed to an equal burden of bacteria throughout the whole trial, which allows for a more straightforward interpretation of the data. The use of killed bacteria is also more elegant from a biosecurity point of view. Because killed instead of alive bacteria were inoculated, healthy animals from a commercial dairy farm could be used, which is different from most other experimental trials in which older culled cows with potentially impaired immunity are often used. In our study, the animals were temporarily extracted from the commercial dairy herd and, after elimination of the infection, again introduced. However, a major drawback of using killed bacteria is that, in general, the host immune responses are less pronounced when killed bacteria are used, thus the observed differences in inflammatory response between vaccinates and nonvaccinates might be underestimated in some cases (Schukken et al., 2011). On the contrary, killed bacteria cannot use their armamentarium to evade or counter the host defenses, which is particularly true for $S$. aureus. Another disadvantage is that the $S$. aureus strain the cows were inoculated with cannot be cultured; thus, PCR-based techniques are required to confirm the presence or absence of the bacteria.

Under equal $S$. aureus burden, the inflammatory reaction was more severe in the nonvaccinated animals than in the vaccinated ones. This corresponds well with the results obtained by Middleton et al. (2006), in which heifers vaccinated with Lysigin, a multivalent whole cell-lysed $S$. aureus bacterin, in late gestation showed less severe clinical symptoms than the nonvaccinated ones after challenge with a heterologous strain of $S$. aureus on d 6, 7, and 8 of lactation. However, in that study, no evidence was found for lower SCC in vaccinates than in nonvaccinates for the tested days; a less pronounced PMN influx as observed in our study for vaccinates does not necessarily imply an impaired immunity. More pronounced host responses might indicate a less efficient reaction, and thus result in a higher likelihood of clinical mastitis. The apparently less severe inflammatory response in the vaccinated animals could explain the lower drop in milk production compared with their nonvaccinated counterparts. Although viable 
milk PMN and generation of reactive oxygen species (ROS) are needed to combat and eliminate invading pathogens, high concentrations during IMI can cause irreversible damage to the udder tissue as well (Burvenich et al., 2003). Interestingly, despite the less severe inflammatory response, bacterial clearance was reached significantly $(P=0.04)$ more often in the vaccinated animals than in the nonvaccinated animals. By way of comparison, no evidence was found by Middleton et al. (2006) for a difference in S. aureus clearance rate $\mathrm{PC}$ between the Lysigin-vaccinated and nonvaccinated dairy heifers. Some cautious is needed in the interpretation of our data, however, as only a semiquantitative method was applied and no strain typing of the isolated S. aureus was performed.

Besides the influx of PMN in the mammary gland, their viability and capacity to generate ROS play a crucial role in the bacterial clearance as well. VandeputteVan Messom et al. (1993) revealed that severe responders to experimentally induced $E$. coli had a lower ROS-generating capacity than moderate responders. In our study, differences between the vaccinated and nonvaccinated animals were observed in neither the prechallenge nor the PC PMN viability. Based on the premise that the viability of PMN is a valid indicator of the ROS-generating capacity, as proposed by Mehrzad et al. (2004), one can assume that another mechanism is involved behind the differences in severity of the inflammatory response to the $S$. aureus challenge between both groups of animals.

An explanation for the less severe, though apparently more efficient, host response in the vaccinates as opposed to the nonvaccinates might be found in the differences in antibody concentration in both serum and whey. Canadian Holsteins with a high antibodymediated immune response, characterized by antibodies of the IgG1 isotype, had less clinical mastitis cases and tended to show less severe clinical symptoms than cows with a low antibody-mediated immune response, suggesting a less severe inflammatory response in case of infection (Thompson-Crispi et al., 2013). Vaccinates and controls had similar SAAC-specific serum antibody concentrations at the time of dry off, before vaccination. Still, at 15 DIM and thus following the double vaccination over the dry period, vaccinates showed higher total antibody titers in both serum and whey as well as higher IgG1 OD, but not of IgG2. The total antibody titers obtained in serum at 15 DIM were slightly higher than those obtained in the study of Prenafeta et al. (2010) in the animals that received a $S$. aureus bacterin with a high SAAC content at the same time after the last vaccination. The IgG1 OD values obtained at dry off, at $10 \mathrm{~d}$ before the expected calving date (i.e., 35 $\mathrm{d}$ after first vaccination), and at 15 DIM were almost identical to those obtained by Prenafeta et al. (2010) in that specific group of animals. Still, the IgG2 OD values in our study remained almost constant over dry period in both the vaccinated and nonvaccinated animals and were lower than those in Prenafeta et al. (2010). Compared with the findings reported by Luby et al. (2007), in which animals were vaccinated with the commercial mastitis vaccine Lysigin containing $S$. aureus bacterin of 1 CP5 strain, 2 CP8 strains, and 2 CP336 strains, the sera SAAC-specific IgG1 OD values corresponded well to the CP8-specific IgG1 titers, which were intermediate to the CP5- and CP336-specific IgG1 titers.

Stimulation of the toll-like receptor 2 (TLR2) by the vaccine is likely what occurred in our study for S. aureus. Typically, TLR2 recognizes lipoteichoic acid and peptidoglycan components from the bacterial cell wall from gram-positive bacteria such as $S$. aureus (Basto and Leitão, 2014). In bovine, TLR2 is mainly expressed on monocytes and monocyte-derived macrophages (Kwong et al., 2011). Activation of the immune responses through TLR2 is not recognized as a strong polarizing stimulus toward either a cell-mediated Thelper(Th)1 or an antibody-mediated Th2 responses, resulting in Th responses with variable characteristics (Basto and Leitão, 2014). In our study, the increase in serum $\operatorname{IgG} 1$ over the dry period, together with the significantly higher IgG1 concentration in whey in vaccinates than in nonvaccinates at both 15 and 17 DIM, indicates a primarily antibody-mediated or Th2 immune response. A similar increase in IgG1 concentration in serum was observed by Luby et al. (2007) in heifers vaccinated in late gestation with Lysigin, though the differences in the whey IgG1 concentration after calving between vaccinates and nonvaccinates were negligible in their study.

Previous contact with $S$. aureus in some animals might hypothetically have hampered the interpretation of the results obtained after in vitro stimulation with $S$. aureus. Still, the high IFN- $\gamma$ levels in some animals at dry off, presumably due to recent previous contact with S. aureus, suggests a cell-mediated Th1 response apart from a Th2 response. The significant increase in IFN- $\gamma$ production in vaccinated animals that presumably had no recent contact with $S$. aureus and the significant difference with their nonvaccinated counterparts also directs toward a moderate Th1 response. However, in contrast to the findings of Luby et al. (2007), no significant changes in IgG2 in serum or whey were observed between vaccinates and nonvaccinates at any of the samplings. Hypothetically, the higher concentrations of IgG1 in vaccinates compared with nonvaccinates might have facilitated phagocytosis of the bacteria via opsonization, which could explain the differences in both new infection and cure rate of $S$. aureus IMI observed 
by Schukken et al. (2014) between both groups kept under the same management conditions. The IgG1 antibodies are in particular good opsonins for macrophages, as the latter leukocytes possess, in contrast to PMN, a large number of $\mathrm{Fc}$ receptors for this subclass (Burton and Erskine, 2003). The IgG1 bound to pathogens can, however, also activate the complement cascade and complement is, in turn, an efficient opsonin for PMN as well.

Vaccinates and nonvaccinates had also similar serum J5-specific total antibody titers and IgG1 and IgG2 OD values at dry off, before the first vaccination. At 15 DIM, just before challenge, the serum total antibody titers were significantly higher in the vaccinated than in the nonvaccinated animals, which corresponds well with previous findings (Wilson et al., 2007; Gurjar et al., 2013). Still, the difference in J5-specific antibody titers between vaccinates and nonvaccinates was not as pronounced as for the SAAC-specific antibodies. Interestingly, some of the nonvaccinates and vaccinates had already J5-specific IgG antibodies in serum at dry off. This suggests that those cows had some previous exposure to coliform pathogens sufficient to mount some immune response, including memory-type responses. Remarkably, no clear switch toward IgG1 or IgG2 was observed. Also, contrasting with Wilson et al. (2007), no significant changes in IgG1 or IgG2 were observed between the vaccinates and nonvaccinates at any of the samplings. Still, the moderate increase in serum IgG2 over the dry period in vaccinates indicates a cell-mediated Th1- response rather than an antibody-mediated or Th2 response. The high IFN- $\gamma$ levels in both vaccinated and nonvaccinated animals at dry off, presumably due to recent previous contact with coliforms, also directs toward a Th1 response. This further substantiated by the more pronounced increase in IFN- $\gamma$ production after in vitro stimulation with $E$. coli over the dry period in those animals that presumably had no recent contact with coliforms. An intramammary challenge with $E$. coli instead of $S$. aureus would most likely have resulted in a substantial increase in IgG2; however, this fell beyond the scope of our study. In whey, no significant differences in total $E$. coli J5 antibody titers were observed between the vaccinated and nonvaccinated animals. Similar to the findings of Wilson et al. (2007), no differences were observed in whey IgG1 nor IgG2 antibody titers at 15 DIM prechallenge between vaccinates and nonvaccinates. The clearly lower response to the J5 than to the SAAC component, as well as the indication for a Th1 response rather than a Th2 response, might at least partly explain why Bradley et al. (2015) only observed significant differences in the severity of the clinical symptoms and not in the clinical mastitis incidence between vaccinates and nonvaccinates.

\section{CONCLUSIONS}

Vaccinated cows had a less-severe inflammatory reaction after inoculation with a killed heterologous $S$. aureus strain compared with nonvaccinated animals. This possibly explains the absence of a substantial drop in milk production in the first days after challenge in the vaccinated animals compared with the nonvaccinated ones. The immune response against $E$. coli after vaccination was less pronounced than the immune response against $S$. aureus. Vaccination with Startvac seems to evoke a strong Th2 response for $S$. aureus, characterized by a shift primarily toward the IgG1 antibody subtype. The type of response against E. coli is less clear, but a Th1 response is suggested based on the weak though significant increase in IgG2 concentrations and the high IFN- $\gamma$ concentrations after in vitro stimulation with E. coli. The increased SAAC-specific antibody concentration in whey in vaccinates compared with nonvaccinates most likely triggers the opsonization of the inoculated $S$. aureus bacteria, apparently resulting in a more efficient immune response and elimination of the bacteria from the mammary gland.

\section{ACKNOWLEDGMENTS}

The authors thank Lydia Bommelé, André Turtelboom, and Karel Vermeulen from Biocentrum AgriVet (Melle, Belgium) for their excellent cooperation and Lars Hulpio from the Mastitis and Milk Quality Research Unit for the excellent technical assistance. This study was financially supported by Laboratorios HIPRA, S.A., (Girona, Spain).

\section{REFERENCES}

Basto, A. P., and A. Leitão. 2014. Targeting TLR2 for vaccine development. J. Immunol. Res. 2014:619410. http://dx.doi. org/10.1155/2014/619410.

Bradley, A. J., J. E. Breen, B. Payne, V. White, and M. J. Green. 2015. An investigation of the efficacy of a polyvalent mastitis vaccine using different vaccination regimens under field conditions in the United Kingdom. J. Dairy Sci. 98:1706-1720.

Burton, J. L., and R. J. Erskine. 2003. Immunity and mastitis-Some new ideas for an old disease. Vet. Clin. North Am. Food Anim. Pract. 19:1-45.

Burvenich, C., V. Van Merris, J. Mehrzad, A. Diez-Fraile, and L. Duchateau. 2003. Severity of $E$. coli mastitis is mainly determined by cow factors. Vet. Res. 34:521-564.

Dufour, S., I. R. Dohoo, H. W. Barkema, L. DesCoteaux, T. J. Devries, K. K. Reyher, J. P. Roy, and D. T. Scholl. 2012. Manageable risk factors associated with the lactational incidence, elimination, and prevalence of Staphylococcus aureus intramammary infections in dairy cows. J. Dairy Sci. 95:1283-1300.

Gurjar, A. A., S. Klaessig, S. A. Salmon, J. Y. Robert, and Y. H. Schukken. 2013. Evaluation of an alternative dosing regimen of a J-5 mastitis vaccine against intramammary Escherichia coli challenge in nonlactating late-gestation dairy cows. J. Dairy Sci. 96:5053-5063. 
Kaesbohrer, A., A. Schroeter, B. A. Tenhagen, K. Alt, B. Guerra, and B. Appel. 2012. Emerging antimicrobial resistance in commensal Escherichia coli with public health relevance. Zoonoses Public Health 59(Suppl. 2):158-165.

Kwong, L. S., R. Parsons, R. Patterson, T. J. Coffey, L. Thonur, J. S. Chang, G. Russell, D. Haig, D. Werling, and J. C. Hope. 2011. Characterisation of antibodies to bovine Toll-like receptor (TLR)2 and cross-reactivity with ovine TLR2. Vet. Immunol. Immunopathol. 139:313-318.

Luby, C. D., J. R. Middleton, J. Ma, C. L. Rinehart, S. Bucklin, C. Kohler, and J. W. Tyler. 2007. Characterization of the antibody isotype response in serum and milk of heifers vaccinated with a Staphylococcus aureus bacterin $\left(\right.$ Lysigin $\left.^{\mathrm{TM}}\right)$. J. Dairy Res. 74:239-246.

Makovec, J. A., and P. L. Ruegg. 2003. Results of milk samples submitted for microbiological examination in Wisconsin from 1994 to 2001. J. Dairy Sci. 86:3466-3472.

Mehrzad, J., L. Duchateau, and C. Burvenich. 2004. Viability of milk neutrophils and severity of bovine coliform mastitis. J. Dairy Sci. $87: 4150-4162$.

Middleton, J. R., J. N. Ma, C. L. Rinehart, V. N. Taylor, C. D. Luby, and B. J. Steevens. 2006. Efficacy of different Lysigin (TM) formulations in the prevention of Staphylococcus aureus intramammary infection in dairy heifers. J. Dairy Res. 73:10-19.

Piccart, K., J. Verbeke, A. De Visscher, S. Piepers, F. Haesebrouck, and S. De Vliegher. 2016. Local host response following an intramammary challenge with Staphylococcus fleurettii and different strains of Staphylococcus chromogenes in dairy heifers. Vet. Res. $47: 56-67$.

Piepers, S., L. De Meulemeester, A. de Kruif, G. Opsomer, H. W. Barkema, and S. De Vliegher. 2007. Prevalence and distribution of mastitis pathogens in subclinically infected dairy cows in Flanders, Belgium. J. Dairy Res. 74:478-483.

Piepers, S., S. De Vliegher, K. Demeyere, B. N. Lambrecht, A. de Kruif, E. Meyer, and G. Opsomer. 2009a. Technical note: Flow cytometric identification of bovine milk neutrophils and simultaneous quantification of their viability. J. Dairy Sci. 92:626-631.

Piepers, S., G. Opsomer, E. Meyer, K. Demeyere, H. W. Barkema, A. de Kruif, and S. De Vliegher. 2009b. Heifer and quarter characteristics associated with periparturient blood and milk neutrophil apoptosis in healthy heifers and in heifers with subclinical mastitis. J. Dairy Sci. 92:4330-4339.
Pitkälä, A., M. Haveri, S. Pyörälä, V. Myllys, and T. Honkanen-Buzalski. 2004. Bovine mastitis in Finland 2001-Prevalence, distribution of bacteria, and antimicrobial resistance. J. Dairy Sci. $87: 2433-2441$.

Prenafeta, A., R. March, A. Foix, I. Casals, and L. Costa. 2010. Study of the humoral immunological response after vaccination with a Staphylococcus aureus biofilm-embedded bacterin in dariy cows: Possible role of the exopolysaccharide specific antibody production in the protection from Staphylococcus aureus induced mastitis. Vet. Immunol. Immunopathol. 134:208-217.

Schukken, Y. H., V. Bronzo, C. Locatelli, C. Pollera, N. Rota, A. Casula, F. Testa, L. Scaccabarozzi, R. March, D. Zalduendo, R. Guix, and P. Moroni. 2014. Efficacy of vaccination on Staphylococcus aureus and coagulase-negative staphylococci intramammary infection dynamics in 2 dairy herds. J. Dairy Sci. 97:5250-5264.

Schukken, Y. H., J. Günther, J. Fitzpatrick, M. C. Fontaine, L. Goetze, O. Holst, J. Leigh, W. Petzl, H.-J. Schuberth, D. G. E. Smith, R. Quesnell, J. Watts, R. Yancey, H. Zerbe, A. Gurjar, R. N. Zadoks, H. -M. Seyfert, and Members of Pfizer Mastitis Research Consortium. 2011. Host-response patterns of intramammary infections in dairy cows. Vet. Immunol. Immunopathol. 144:270-289.

Thompson-Crispi, K. A., F. Miglior, and B. A. Mallard. 2013. Incidence rates of clinical mastitis among Canadian Holsteins classified as high, average, or low immune responders. Clin. Vaccine Immunol. 20:106-112.

Thrusfield, M., C. Ortega, I. de Blas, J. P. Noordhuizen, and F. Frankena. 2001. WIN EPISCOPE 2.0: improved epidemiological software for veterinary medicine. Vet. Rec. 148:567-572.

Van Oostveldt, K., F. Vangroenweghe, H. Dosogne, and C. Burvenich. 2001. Apoptosis and necrosis of blood and milk polymorphonuclear leukocytes in early and midlactating healthy cows. Vet. Res. 32:617-622.

Vandeputte-Van Messom, G., C. Burvenich, E. Roets, A. M. MassartLeën, R. Heyneman, W. D. Kremer, and A. Brand. 1993. Classification of newly calved cows into moderate and severe responders to experimentally induced Escherichia coli mastitis. J. Dairy Res. 60:19-29.

Wilson, D. J., B. A. Mallard, J. L. Burton, Y. H. Schukken, and Y. T. Gröhn. 2007. Milk and serum J5-specific antibody responses, milk production change, and clinical effects following intrammary Escherichia coli challenge for J5 vaccinate and control cows. Clin. Vaccine Immunol. 14:693-699. 